ARTICLE

\title{
Scalable salt-templated synthesis of two-dimensional transition metal oxides
}

Xu Xiao ${ }^{1, \star}$, Huaibing Song ${ }^{1, \star}$, Shizhe Lin ${ }^{1}$, Ying Zhou ${ }^{1}$, Xiaojun Zhan ${ }^{1}$, Zhimi Hu${ }^{1}$, Qi Zhang ${ }^{2}$, Jiyu Sun ${ }^{1}$, Bo Yang $^{1}$, Tianqi $\mathrm{Li}^{1}$, Liying Jiao ${ }^{2}$, Jun Zhou ${ }^{1}$, Jiang Tang $^{1} \&$ Yury Gogotsi ${ }^{3}$

Two-dimensional atomic crystals, such as two-dimensional oxides, have attracted much attention in energy storage because nearly all of the atoms can be exposed to the electrolyte and involved in redox reactions. However, current strategies are largely limited to intrinsically layered compounds. Here we report a general strategy that uses the surfaces of water-soluble salt crystals as growth templates and is applicable to not only layered compounds but also various transition metal oxides, such as hexagonal- $\mathrm{MoO}_{3}, \mathrm{MoO}_{2}, \mathrm{MnO}$ and hexagonal$\mathrm{WO}_{3}$. The planar growth is hypothesized to occur via a match between the crystal lattices of the salt and the growing oxide. Restacked two-dimensional hexagonal- $\mathrm{MoO}_{3}$ exhibits high pseudocapacitive performances (for example, $300 \mathrm{~F} \mathrm{~cm}^{-3}$ in an $\mathrm{Al}_{2}\left(\mathrm{SO}_{4}\right)_{3}$ electrolyte). The synthesis of various two-dimensional transition metal oxides and the demonstration of high capacitance are expected to enable fundamental studies of dimensionality effects on their properties and facilitate their use in energy storage and other applications.

\footnotetext{
${ }^{1}$ Wuhan National Laboratory for Optoelectronics and School of Optical and Electronic Information, Huazhong University of Science and Technology, Wuhan, Hubei 430074, China. ${ }^{2}$ Key Lab of Organic Optoelectronics \& Molecular Engineering, Department of Chemistry, Tsinghua University, Beijing 100084, China. ${ }^{3}$ Department of Materials Science and Engineering and A.J. Drexel Nanomaterials Institute, Drexel University, Philadelphia, Pennsylvania 19104, USA.

* These authors contributed equally to this work. Correspondence and requests for materials should be addressed to J.Z. (email: jun.zhou@mail.hust.edu.cn) or to J.T. (email: jtang@mail.hust.edu.cn) or to Y.G. (email: gogotsi@drexel.edu).
} 
$\mathrm{P}$ seudocapacitors are of great interest for energy storage owing to their fast charging/discharging, high power density and excellent cycling stability, which are beneficial to many potential applications ranging from ubiquitous portable electronics to grid energy storage ${ }^{1-7}$. Recently, the synthesis of two-dimensional (2D) nanosheets followed by restacking of these nanosheets to form electrodes has attracted much attention ${ }^{8-11}$. Because of their nanometre/sub-nanometre thickness, nearly all of the metal atoms can be exposed to the electrolyte and potentially involved in redox reactions, resulting in pseudocapacitances that approach the theoretical values ${ }^{9}$. Prominent examples of 2D nanosheets for pseudocapacitor electrodes include MXenes, which have exhibited a volumetric capacitance as high as $900 \mathrm{~F} \mathrm{~cm}^{-3}$ owing to their high conductivity and hydrophilicity, and $1 \mathrm{~T}$ phase $\mathrm{MoS}_{2}$ nanosheets, which have exhibited a volumetric capacitance of $650 \mathrm{~F} \mathrm{~cm}^{-1}$ (refs 3,9,11).

Transition metal oxides, such as $\mathrm{MnO}_{2}$ and $\mathrm{RuO}_{2}$ are classic pseudocapacitor materials that possess a large theoretical capacity owing to their large number of oxidation states ${ }^{12-14}$. Moreover, oxides have a higher chemical stability than sulfides, carbides and nitrides, such as $\mathrm{MoS}_{2}$ (ref. 11) or MXenes ${ }^{9-10}$, especially when processed into $2 \mathrm{D}$ flakes, which may provide a better durability and enable use in positive electrodes. In addition, many oxides contain tunnels or pores within the crystal structure, which is also beneficial for energy storage applications ${ }^{2,3}$. Despite the recent demonstration of promising energy application of nanometrethick liquid-exfoliated $\alpha-\mathrm{MoO}_{3}$ (ref. 15) and $\mathrm{MnO}_{2}$ (refs 12,14), the investigation of many other oxides for pseudocapacitor applications is hindered by their lack of a layered structure. Therefore, production of a $2 \mathrm{D}$ morphology is challenging.

For pseudocapacitor applications, nanosheets are highly preferable because ion diffusion within the oxide lattice is minimized, enabling the simultaneous achievement of a large volumetric capacitance (high energy density) at a high rate (high power density). This behaviour is typically very challenging to achieve for both bulk and one-dimensional materials. Ideally, the synthesized 2D oxide should be free of contamination from intercalating chemicals, dispersing agents and surfactants that could compromise its electrochemical performance owing to possible blockage of active sites and the added weight/volume of electrochemically inactive contaminants. In addition, the synthesis should be scalable for industrial manufacturing and production of large-size nanosheets with tunable compositions (for example, oxygen vacancies) to compensate for the poor conductivity often encountered in oxides. The current strategies for the production of 2D materials include gas-phase and solution syntheses. Gas-phase synthesis including chemical vapour deposition $^{16-18}$ can produce large-size and high-quality $2 \mathrm{D}$ nanosheets on a substrate, but is hindered by a low yield. Solution-based processes, such as liquid exfoliation ${ }^{12,15,19-21}$ or chemical synthesis ${ }^{22-26}$, are simple and scalable. However, these processes lead to small-size particles and have a higher probability of contamination from the synthetic process. One additional limit for exfoliation is its restriction to layered compounds. Only materials bonded by van der Waals or other weak forces in one direction can be easily cleaved by the intercalants. Therefore, the development of a general and scalable strategy for the synthesis of high-quality 2D oxides, including those with non-layered structures and investigation of their potential for pseudocapacitor applications are important.

Herein, we report a general strategy that combines many of the advantages of vacuum-based and solution-based synthetic methods that can be applied to various oxides, including non-layered compounds. Synthesized 2D hexagonal- $\mathrm{MoO}_{3}(h-$ $\left.\mathrm{MoO}_{3}\right)$ is shown to be $300 \mathrm{~F} \mathrm{~cm}^{-3}$ at $5 \mathrm{mV} \mathrm{s}^{-1}$ in $\mathrm{Al}_{2}\left(\mathrm{SO}_{4}\right)_{3}$ electrolyte and approaches the theoretical capacitance of the material at a charge/discharge rate of $2 \mathrm{mV} \mathrm{s}^{-1}$ in organic electrolyte. For the 2D oxide synthesis, a molecular precursor solution was first prepared and mixed with a large volume of an inorganic salt (Fig. 1a,b, and Supplementary Fig. 1 shows scanning electron microscopy (SEM) images of precursor@salt templates). Next, the solution was dried and the mixture was annealed at an elevated temperature to produce $2 \mathrm{D}$ oxides, through an intermediate hydroxide phase in some cases (Supplementary Fig. 2). The salts were dissolved and the nanosheets were assembled into a restacked film after rinsing and filtration (Fig. 1c,d). In this process, the salt microcrystals served as the substrate/template to guide the oxide growth at elevated temperatures. The as-produced $2 \mathrm{D}$ oxides exhibited a large lateral size (up to $100 \mu \mathrm{m}$ ), which is an advantage of a vacuum-based synthesis approach. The process can be scaled up by increasing the amount of inexpensive salt used, as evidenced by the large amount of precursors and final products shown in Fig. 1e and Supplementary Fig. 3. The salt can be dissolved in water, leaving behind clean products that can be further directly filtered into binder- and additive-free films for pseudocapacitor electrodes and other applications (Fig. 1d,f). When integrated into an electrochemical device, ions can easily diffuse from the electrolyte into the restacked film to react with the surface active sites, which are fully exposed in these atomically thin 2D oxides. Under these circumstances, the rate-limiting step of ion intercalation and diffusion in bulk materials is largely circumvented, and all of the active material participates in redox reactions, which contribute to electrochemical energy storage.

\section{Results}

Synthesis of 2D oxides. Using this strategy, several 2D oxides, including $h-\mathrm{MoO}_{3}, h-\mathrm{WO}_{3}, \mathrm{MoO}_{2}$, and $\mathrm{MnO}$ were successfully synthesized as shown in Fig. 2. On the basis of the SEM and optical images (Supplementary Fig. 4), all of the samples possess 2D morphology with the size of some flakes exceeding $400 \mu \mathrm{m}^{2}$, which is much larger than that of liquid-exfoliated or chemically synthesized samples in which sizes are typically limited to a few micrometres ${ }^{27}$. The dimensions of the salt crystals are tens of micrometres and guide the growth of the $2 \mathrm{D}$ oxide to a similar size. The transmission electron microscopy analysis of these oxides (Fig. 2e-h; Supplementary Fig. 5) further confirmed the planar 2D morphology, and the high transparency and observed wrinkles suggest that the as-synthesized oxides are nanosheets. All of the oxide crystals had a thickness of $<2 \mathrm{~nm}$, which was determined using atomic force microscopy (AFM) (Fig. 2i-l; Supplementary Fig. 6). Selected area electron diffraction, X-ray diffraction (Supplementary Fig. 7) and X-ray photoelectron spectroscopy (XPS; Supplementary Figs 8 and 9) confirmed the formation of $h-\mathrm{MoO}_{3}, h-\mathrm{WO}_{3}$, monoclinic $\mathrm{MoO}_{2}$ and cubic $\mathrm{MnO}$. Moreover, X-ray diffraction characterization also revealed broadened diffraction peaks corresponding to $2 \mathrm{D} \mathrm{MoO}_{2}$ that were shifted to small angles compared with those of bulk $\mathrm{MoO}_{2}$, suggesting lattice relaxation in the $2 \mathrm{D} \mathrm{MoO}_{2}$ (Supplementary Fig. 10). On the basis of the naturally non-layered crystal structures of these oxides (Supplementary Fig. 11), the suitability of salt-assisted templating as a general method for the synthesis of $2 \mathrm{D}$ oxides has been convincingly demonstrated. Despite previous studies using $\mathrm{NaCl}$ to synthesize oxide nanocubes ${ }^{28-30}$, to the best of our knowledge, the use of inorganic salts to synthesize $2 \mathrm{D}$ oxides has not been previously reported.

Growth mechanism. Next, we will discuss the synthesis of $\mathrm{MnO}$, which has a non-layered cubic structure (Supplementary Fig. 11d). First, $\mathrm{Mn}\left(\mathrm{CH}_{3} \mathrm{COO}\right)_{2}$, which was dissolved in ethanol, was used as the molecular precursor to avoid nucleation 


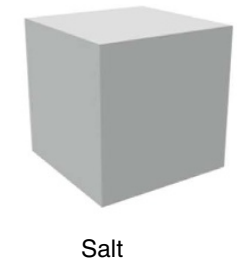

d

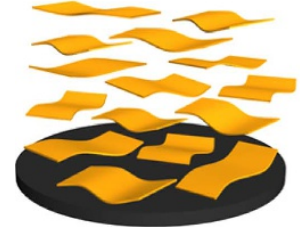

b

Precursor@salt

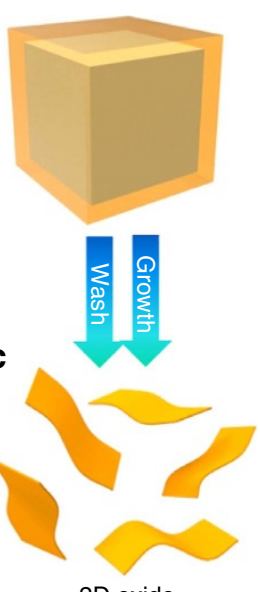

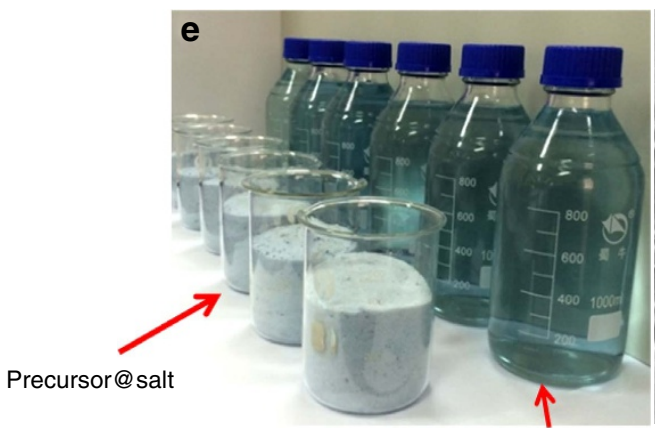

2D oxide

2D oxide dispersion

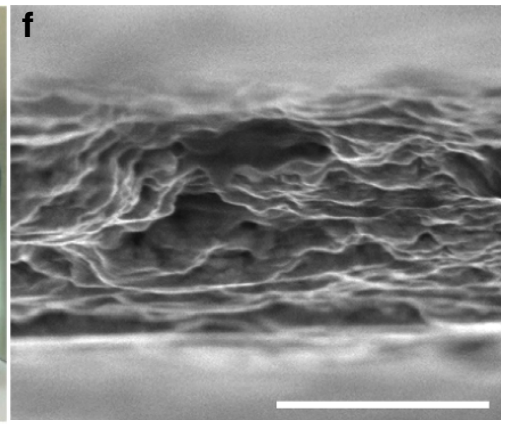

Figure 1 | Schematic representation of $\mathbf{2 D} \mathbf{h}-\mathbf{M o O}_{3}$ synthesis and electrode fabrication for energy storage. (a,b) Schematic representation of the fabrication process. The precursor solution was coated on the salt by mixing the solution with a large amount of salt microcrystals. (c) After thermal

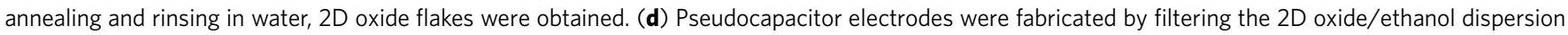
using a Celgard separator. (e) Optical image of the precursors and 2D $h$ - $\mathrm{MoO}_{3}$-ethonal dispersions. The front row shows six beakers containing the precursor, and the back row shows six bottles containing the $2 \mathrm{D} h-\mathrm{MoO}_{3}$-ethanol dispersions. (f) Multilayered $2 \mathrm{D}$ oxide is shown in the cross-sectional SEM image. Scale bar, $1 \mu \mathrm{m}$.

of oxide nanocrystals in the solution. Second, the thickness of the $2 \mathrm{D}$ oxide was controlled by mixing a limited volume of the dilute oxide precursor solution with a large quantity of salt microcrystals. Using this approach, the $2 \mathrm{D}$ oxide is believed to be confined to a monolayer thickness, and the formation of structurally stable nanocubes ${ }^{31-34}$ was avoided because of an insufficient amount of precursor and large-size salt crystals. We measured the size of the salt microcrystals and performed calculations of the precursor-to-salt ratio to meet this requirement (Supplementary Fig. 12; Supplementary Note 1). The AFM analysis indicated that the as-produced $2 \mathrm{D} \mathrm{MnO}$ crystals were $\sim 1.3 \mathrm{~nm}$ thick (Supplementary Fig. $6 \mathrm{~d}$ ). Third, the crystal geometry of the substrate (salt) should be identical to the target oxide to promote Frank-van der Merwe mode film growth and possible heteroepitaxy ${ }^{35,36}$. MnO was cubic with a lattice constant $\left(a_{1}\right)$ of $0.4442 \mathrm{~nm}$. Therefore, $\mathrm{KCl}$ was chosen as the template because of its similar cubic structure with a lattice constant $\left(a_{2}\right)$ of $0.3138 \mathrm{~nm}$. Because the crystal symmetry was identical and $\sqrt{ } 2 a_{2}$ was $0.4437 \mathrm{~nm}$, overlap of the atomic configuration could be achieved by rotating the crystal plane of $\mathrm{MnO}$ by $45^{\circ}$. Figure 3 a shows the (001) crystal plane of $2 \mathrm{D} \mathrm{MnO}$ on the (001) plane of $\mathrm{KCl}$. The mismatch between $\mathrm{KCl}$ and $\mathrm{MnO}$ is only $0.11 \%$ (Supplementary Fig. 13). The X-ray diffraction pattern of the mixture of $\mathrm{KCl}$ salt and 2D MnO (after annealing but before rinsing) indicated close pairing of the (200) and (220)

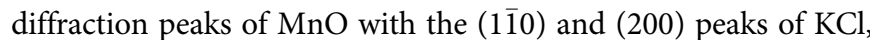
confirming our hypothesis (Fig. 3b). The importance of lattice matching is supported by two facts. First, when $\mathrm{Na}_{2} \mathrm{SO}_{4}$ was chosen as the template salt, no $2 \mathrm{D} \mathrm{MnO}$ was produced using the same preparation procedure because of the large lattice mismatch between $\mathrm{MnO}$ and $\mathrm{Na}_{2} \mathrm{SO}_{4}$ (Supplementary Figs $13 \mathrm{e}$ and 14). Second, when we directly dropped the precursor solution onto the ceramic boat (no template) and annealed it under the same conditions, particles were produced rather than 2D structures (Supplementary Fig. 15a,b).

The same approach was used to synthesize $2 \mathrm{D} h-\mathrm{MoO}_{3}$. Mo was dissolved in $\mathrm{H}_{2} \mathrm{O}_{2}$ to produce the molecular peroxomolybdate precursor solution. A precisely calculated amount of precursor was loaded to restrict the growth of thick nanosheets, and $\mathrm{NaCl}$ microcrystals were used as lattice-matched templates. Similar close pairing of the diffraction peaks between $h-\mathrm{MoO}_{3}$ $((1 \overline{4} 0)$ peak) and the $\mathrm{NaCl}$ templates $((2 \overline{2} 0)$ peak; cubic $a=5.62 \AA)$ was also observed in the X-ray diffraction patterns (Fig. 3d), and the $\mathrm{MoO}_{3}$ crystal growth on $\mathrm{NaCl}$ is shown in the inset and in Fig. 3c. As previously suggested in the literature ${ }^{37}$, the formation of $h-\mathrm{MoO}_{3}(a=10.584 \AA, c=3.7278 \AA)$ rather than $\alpha-\mathrm{MoO}_{3}$ is most likely due to $\mathrm{Na}^{+}$serving as a mineralizer and stabilizer of the hexagonal tunnels in $h-\mathrm{MoO}_{3}$ in addition to the lattice match-induced $h-\mathrm{MoO}_{3}$ nucleation. It is important to note that amorphous $\mathrm{MoO}_{3}$ particles were obtained when no template 

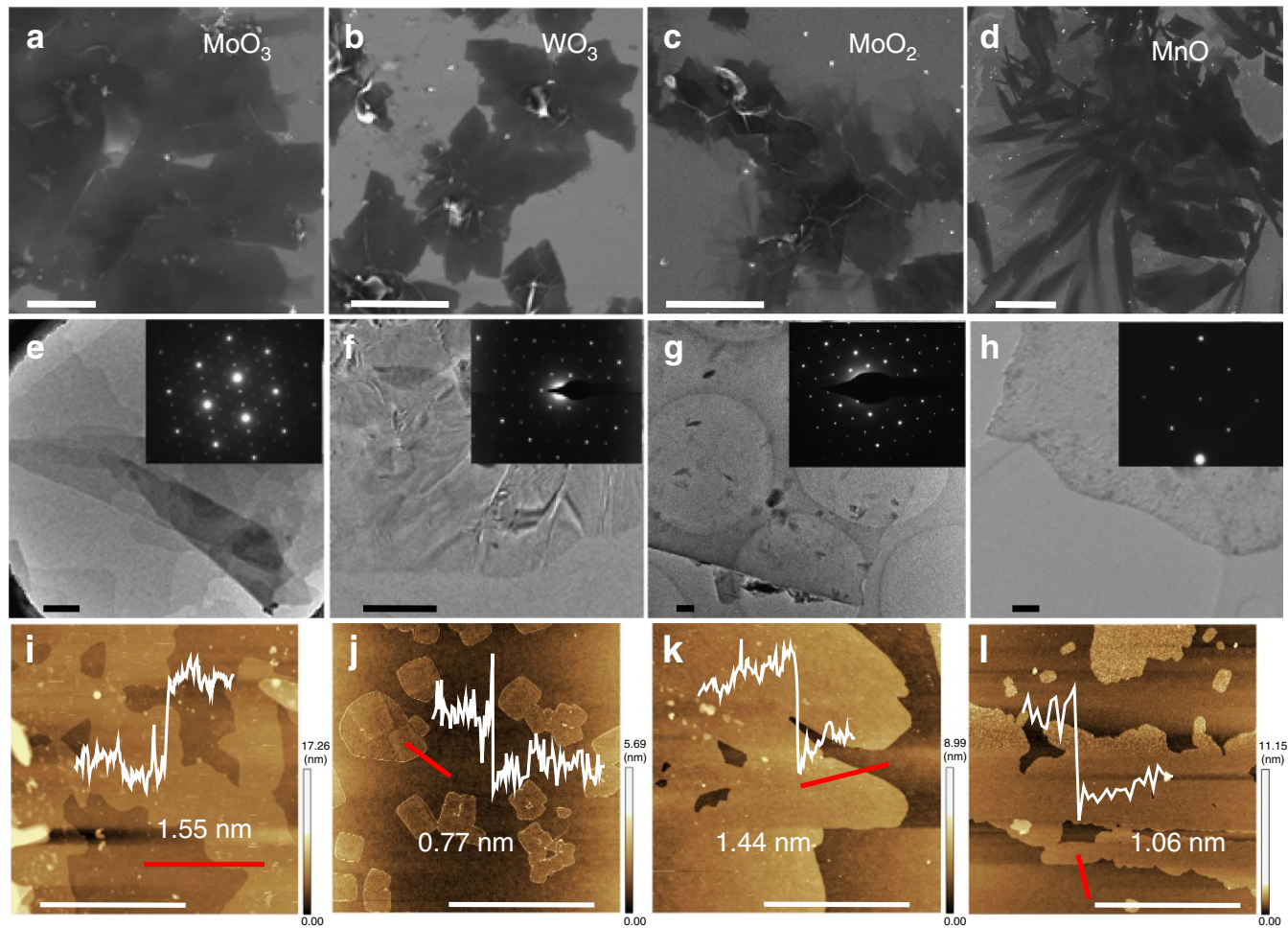

Figure 2 | High-quality 2D oxide synthesized using the salt-assisted templating method. SEM images of $h-\mathrm{MoO} 3$ (a) $h$-WO3 (b) $\mathrm{MoO} 2$ (c) and $\mathrm{MnO}$ (d). Scale bar, $20 \mu \mathrm{m}(\mathbf{a}, \mathbf{b}) ; 100 \mu \mathrm{m}$ (c); $50 \mu \mathrm{m}$ (d). Low-resolution TEM images of $h$-MoO3 (e), $h$-WO3 (f), MoO2 (g) and MnO (h). Scale bar, $500 \mathrm{~nm}$ (for all of the TEM images). The insets show the corresponding selected area electron diffraction patterns. AFM images of $h$-MoO3 (i), $h$-WO3 (j), MoO2 (k) and $\mathrm{MnO}(\mathbf{I})$. The insets show the corresponding thickness of the 2D oxide. Scan lines are shown in red. Scale bar, $1 \mu \mathrm{m}(\mathbf{i}, \mathbf{k}) ; 5 \mu \mathrm{m}(\mathbf{j}, \mathbf{I})$.

a

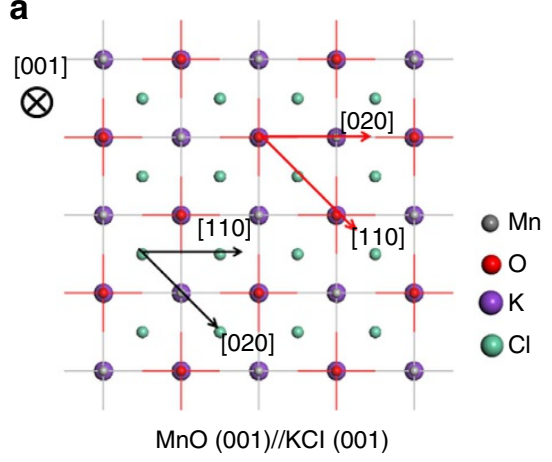

C

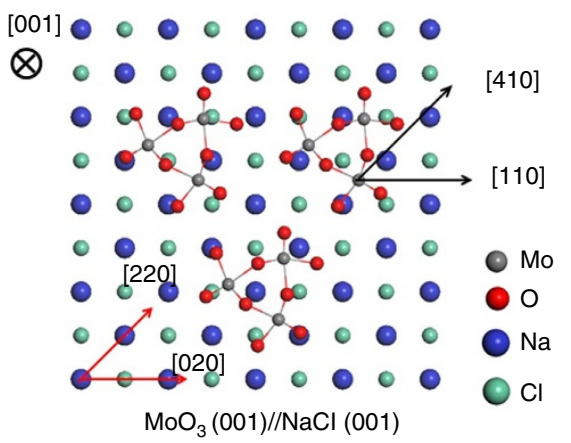

b

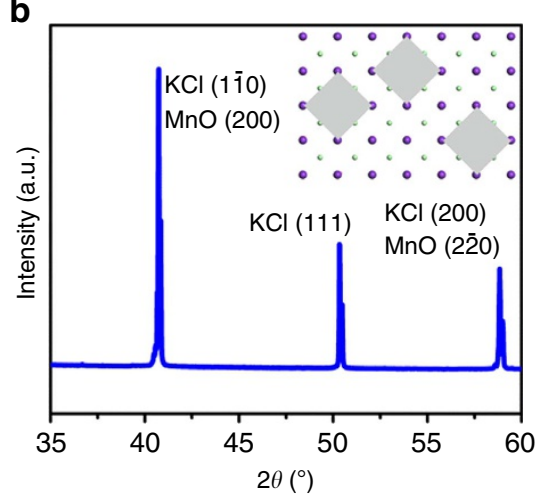

d

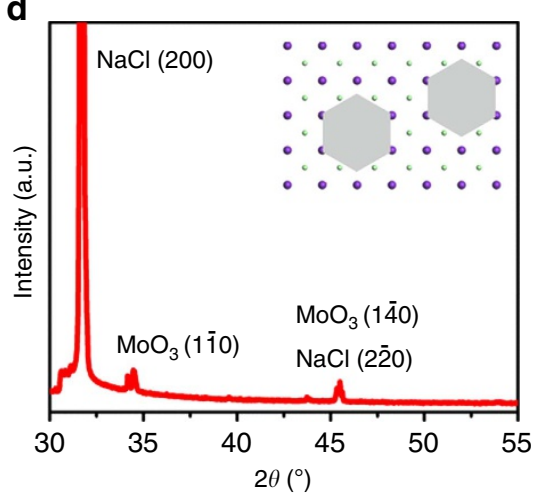

Figure 3 | Proposed mechanism of the salt-assisted templated growth of 2D $\mathbf{M n O}$ and $\boldsymbol{h}-\mathbf{M o O}_{\mathbf{3}}$. (a) Top view of the crystal modes of the MnO (001) plane sitting on the $\mathrm{KCl}(001)$ plane. (b) X-ray diffraction pattern of $2 \mathrm{D} \mathrm{MnO}$ on $\mathrm{KCl}$. Please note that the faint diffraction peaks of $\mathrm{MnO}$ reside at the left side of $\mathrm{KCl}$ diffraction peaks. The inset is a schematic representation of cubic $\mathrm{MnO}$ on $\mathrm{KCl}$. (c) Top view of the crystal modes of the $h-\mathrm{MoO}_{3}(001)$ plane sitting on the $\mathrm{NaCl}$ (001) plane. (d) X-ray diffraction pattern of $2 \mathrm{D} h-\mathrm{MoO}_{3}$ on $\mathrm{NaCl}$. The inset is a schematic representation of $h-\mathrm{MoO}{ }_{3}$ on $\mathrm{NaCl}$. 
was used, further demonstrating the importance of the presence of $\mathrm{NaCl}$ (Supplementary Fig. 15c,d). Therefore, we hypothesize that the lateral growth of oxides on the salt surfaces is guided by the salt crystal geometry and promoted by lattice matching, and the growth perpendicular to the salt surface is limited by precursor depletion, which leads to the synthesis of oxide nanosheets with nanometre thickness even for materials with a non-layered crystal structure. In addition, the thickness can be easily controlled by tuning the precursor-to-salt ratio. The thickness increased from 1.6 to $\sim 12 \mathrm{~nm}$ when the precursorto-salt ratio was increased by a factor of 8 (Supplementary Fig. 16). Nonetheless, more characterizations are required to completely elucidate the mechanism of nanosheet growth on salt templates.

Electrochemical performance of $\mathbf{2 D} \boldsymbol{h}-\mathbf{M o O}_{3}$. To explore the electrochemical performance of a $2 \mathrm{D}$ oxide, $2 \mathrm{D} h-\mathrm{MoO}_{3}$ was selected because $\mathrm{MoO}_{3}$ possesses a high theoretical capacity $(1.5 \mathrm{Li} / \mathrm{Mo})^{38}$. Notably, this high theoretical capacity is only achievable at a very low current density $\left(0.01 \mathrm{Ag}^{-1}\right)$ and a slow charging/discharging rate, which is not suitable for electrochemical capacitors ${ }^{2}$. When processed into $2 \mathrm{D}$ nanosheets, we expected that the confinement by slow ion diffusion in bulk $\mathrm{MoO}_{3}$ would be relaxed, which would enable fast and reversible surface reactions to achieve high capacitance at high rates to allow for the use of $\mathrm{MoO}_{3}$ in pseudocapacitor applications ${ }^{39}$. Using a three-electrode configuration, six different aqueous electrolytes were explored with different cation sizes (Supplementary Fig. 17a,b), and the results are summarized in Fig. 4a. The highest volumetric capacitance of $600 \mathrm{~F} \mathrm{~cm}^{-3}$ was achieved in $\mathrm{H}_{2} \mathrm{SO}_{4}$ at $5 \mathrm{mV} \mathrm{s}^{-1}$, which is larger than the value for the $\mathrm{Ti}_{3} \mathrm{C}_{2} \mathrm{~T}_{x}$ paper in $\mathrm{KOH}\left(\sim 400 \mathrm{~F} \mathrm{~cm}^{-3}\right)^{10}$ and chemically converted graphene in $\mathrm{H}_{2} \mathrm{SO}_{4}\left(256 \mathrm{~F} \mathrm{~cm}^{-3}\right)^{8}$. All of the results indicated superior rate capability, which is crucial for pseudocapacitors. Surprisingly, the performance in the $\mathrm{Al}_{2}\left(\mathrm{SO}_{4}\right)_{3}$ electrolyte was $300 \mathrm{~F} \mathrm{~cm}^{-3}$ at $5 \mathrm{mV} \mathrm{s}^{-1}$, and to the best of our knowledge, this high capacitance in an Al salt electrolyte has not been previously reported for any material, which opens the door for the use of $2 \mathrm{D}$ oxides in Al-ion batteries ${ }^{40}$. Furthermore, the electrochemical impedance spectroscopy results shown in Fig. $4 \mathrm{~b}$ indicate that the $2 \mathrm{D}$ $h-\mathrm{MoO}_{3}$ electrode exhibited good capacitive behaviour with no apparent charge transfer resistance, which indicates excellent ionic and electronic conductivity. The enhanced electron conductivity arises from the large dimension (fewer and better contact for each flake in the restacked electrode) and abundant oxygen vacancies present in our $2 \mathrm{D} h-\mathrm{MoO}_{3}$ (XPS, Raman spectroscopy, photoluminescence characterizations and conductivity measurements; Supplementary Figs 8a, 18, 19 and 20). These properties are intrinsic advantages of the proposed synthesis strategy where the large size is inherited from the $\mathrm{NaCl}$ microcrystal templates and the oxygen vacancies originated from thermal annealing at elevated temperatures in an oxygenfree atmosphere. In addition, a control experiment using $\sim 12 \mathrm{~nm}$ $h-\mathrm{MoO}_{3}$ nanosheets (Supplementary Fig. 17c,d) resulted in a significantly decreased volumetric capacitance compared with that of the $1.6 \mathrm{~nm} h-\mathrm{MoO}_{3}$ at the same sweep rate, further confirming the effectiveness of nanometre-thin $2 \mathrm{D} h-\mathrm{MoO}_{3}$ to minimize the restriction of ion diffusion ${ }^{41}$. In addition, the electrochemical performance of the $\mathrm{MoO}_{3}$ particles that were obtained without templates (Supplementary Fig. 21) was investigated, and a capacitance of $<50 \mathrm{~F} \mathrm{~cm}^{-3}$ was achieved, which is significantly lower than that for $2 \mathrm{D} h-\mathrm{MoO}_{3}$ and demonstrates the advantage of the $2 \mathrm{D}$ morphology.

Although aqueous electrolytes are widely used, they are inferior to organic electrolytes in terms of the energy density owing to the narrow potential window, which is limited by water splitting. To maximize the energy stored in $2 \mathrm{D} h-\mathrm{MoO}_{3}, 2 \mathrm{D} h-\mathrm{MoO}_{3}$ was tested in a $1 \mathrm{M} \mathrm{LiClO}_{4}$ solution with a 1:1 mixture of ethylene carbonate and dimethyl carbonate. The contact angle measurements indicate that the organic electrolyte provides good wetting of the restacked 2D $h-\mathrm{MoO}_{3}$ electrode film (Supplementary Fig. 22). According to the cyclic voltammetry (CV) curves at different sweep rates (Fig. 5a), a pair of wide peaks arising from reduction/oxidation reactions contributed to the pseudocapacitance $^{41}$. The charge-discharge curves at different current densities also indicate pseudocapacitive behaviour (Supplementary Fig. 23a). The volumetric and gravimetric capacities are shown in Fig. 5b within a charging/discharging time that ranged from 10 to 1,000 s. Intriguingly, a gravimetric capacity of $996 \mathrm{Cg}^{-1}\left(277 \mathrm{mAhg}^{-1}\right)$ at $2 \mathrm{mV} \mathrm{s}^{-1}$ was much larger than that previously reported for mesoporous $\alpha-\mathrm{MoO}_{3}$ in $1 \mathrm{M} \mathrm{LiClO} 4$ with propylene carbonate $\left(\sim 580 \mathrm{Cg}^{-1}\right.$, $\left.161 \mathrm{mAhg}^{-1}\right)^{41}$. When the charging/discharging time was limited to $10 \mathrm{~s}$, the capacity was still $400 \mathrm{Cg}^{-1}$, suggesting that the $2 \mathrm{D} h-\mathrm{MoO}_{3}$ electrode can provide excellent power handling that is suitable for quick charging of portable or wearable electronics.

The redox reaction can be expressed as follows:

$$
\mathrm{MoO}_{3}+x \mathrm{Li}^{+}+x \mathrm{e}^{-} \longleftrightarrow \mathrm{Li}_{x} \mathrm{MoO}_{3}
$$

Because the charge of an electron is identical to a lithium ion, the number of $\mathrm{Li}^{+}$involved in the redox reaction can be determined as follows: $x=Q M / m F$, where $Q$ is the stored charge, $M$ is the molecular weight, $m$ is the mass and $F$ is the Faraday constant. As
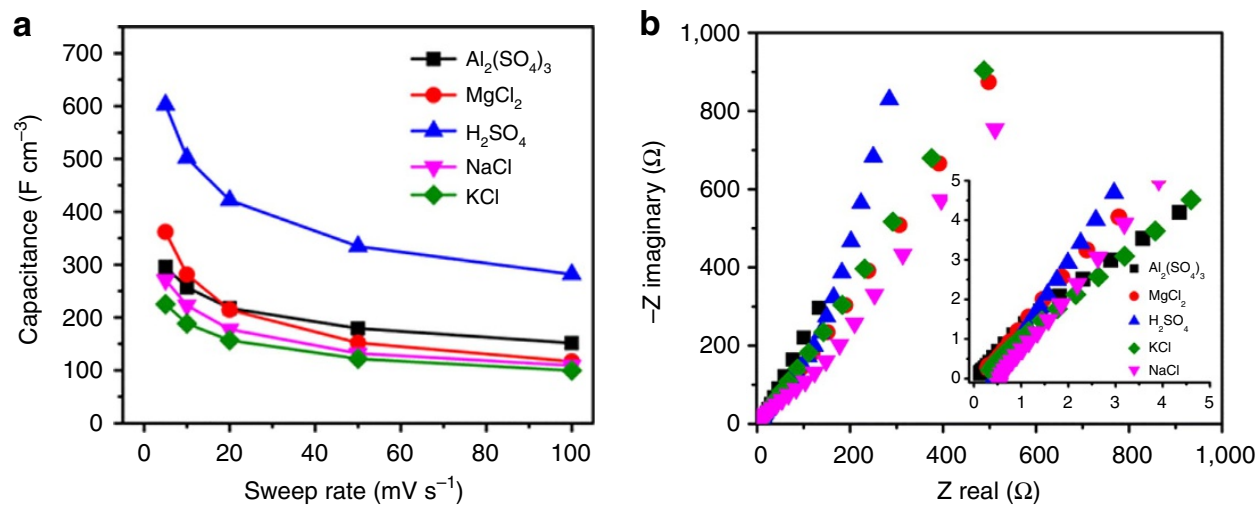

Figure 4 | Electrochemical performance of 2D $\mathbf{h}-\mathbf{M o O}_{3}$ in aqueous electrolytes. (a) Volumetric capacitance as a function of the sweep rate in different aqueous electrolytes. (b) Nyquist plots obtained in $\mathrm{H}_{2} \mathrm{SO}_{4}, \mathrm{MgCl}_{2}, \mathrm{NaCl}, \mathrm{KCl}$ and $\mathrm{Al}_{2}\left(\mathrm{SO}_{4}\right)_{3}$ electrolytes. 

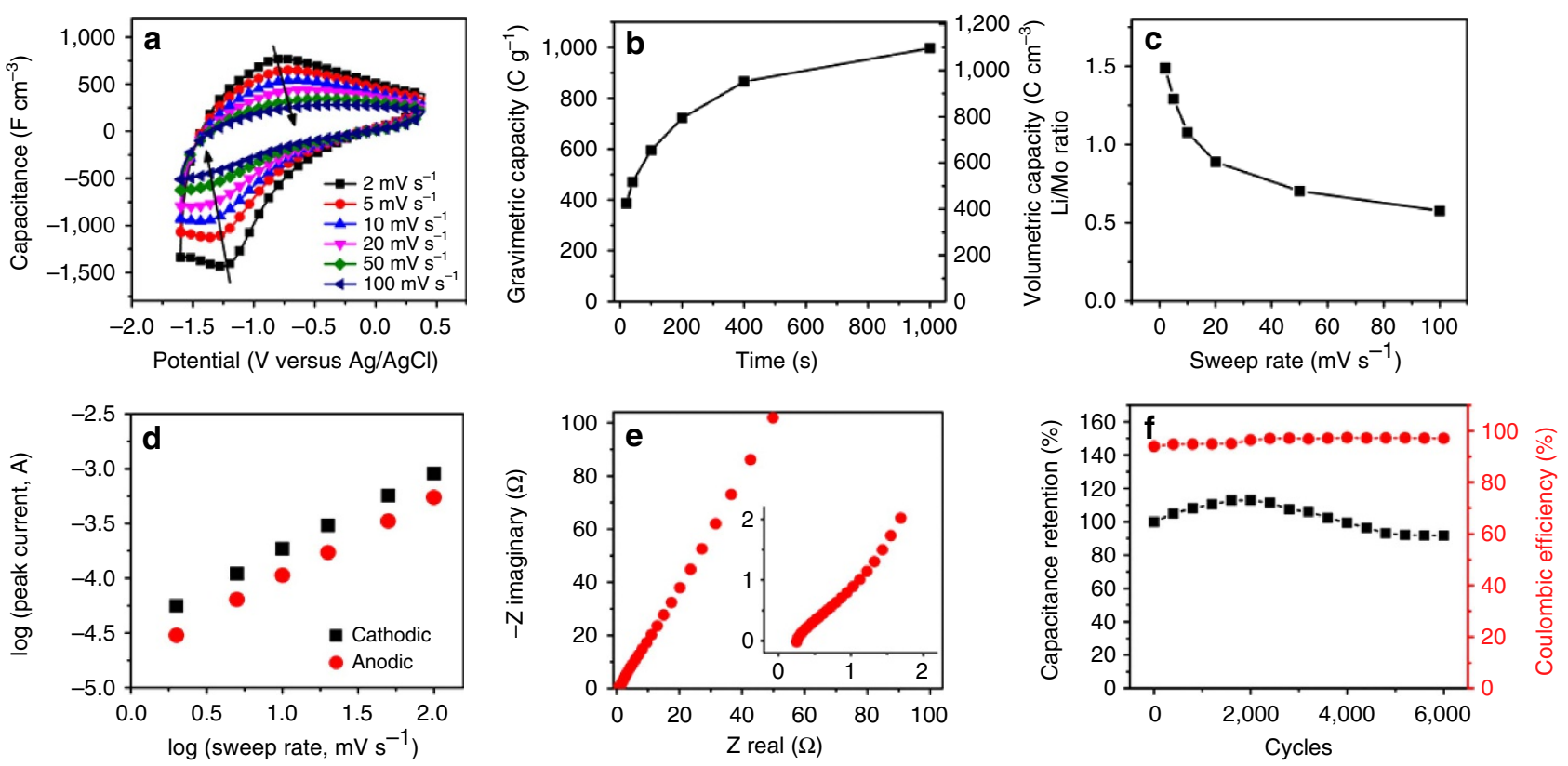

Figure $\mathbf{5}$ | Electrochemical performance of $\mathbf{2 D} \mathbf{h}-\mathbf{M o O}_{\mathbf{3}}$ in a Li-ion-containing organic electrolyte. (a) $\mathrm{CV}$ curves at sweep rates ranging from 2 to $100 \mathrm{mVs}^{-1}$ indicating the small peak shifts following the arrows. (b) Gravimetric and volumetric capacity as a function of charging/discharging time. (c) Li/Mo ratio at different sweep rates using $x=Q M / m F$, where $Q$ is the stored charge, $M$ is the molecular weight, $m$ is the mass and $F$ is the Faraday constant. After $1,000 \mathrm{~s}, 2 \mathrm{D} \mathrm{h}-\mathrm{MoO}_{3}$ reaches $1.49 \mathrm{Li} / \mathrm{Mo}$, approaching the theoretical value for bulk $\alpha-\mathrm{MoO}_{3}$ (1.5). (d) Plot for calculating $b$ values of the cathodic and anodic peaks (both $=0.9$ ). (e) Nyquist plots. (f) Cycling stability and Coulombic efficiency over 6,000 cycles.

shown in Fig. 5c, $1.49 \mathrm{Li}^{+}$per $\mathrm{MoO}_{3}$ were inserted at $2 \mathrm{mV} \mathrm{s}^{-1}$ and $0.58 \mathrm{Li} / \mathrm{Mo}$ were inserted at $100 \mathrm{mV} \mathrm{s}^{-1} \cdot \alpha-\mathrm{MoO}_{3}$ can accommodate up to $1.5 \mathrm{Li} / \mathrm{Mo}$ at a very low current density $\left(0.01 \mathrm{~A} \mathrm{~g}^{-1}\right)$, and this amount is largely limited to the surface charge storage at high rates. For bulk and even nanostructured materials with thicknesses greater than the atomic level, slow ion diffusion in solids limits the storage capacity and device power ${ }^{1}$. Restacked $2 \mathrm{D} h-\mathrm{MoO}_{3}$ exhibited a different behaviour because the entire active material was accessible to the electrolyte, allowing for easy $\mathrm{Li}^{+}$transport to the surface that facilitated surface redox reactions with the exposed transition metal atoms and led to a high capacitance even at high sweep rates, as shown in Fig. 1e. Therefore, this high capacitance approaches the theoretical capacitance at $2 \mathrm{mV} \mathrm{s}^{-1}$, confirming the advantage of $2 \mathrm{D}$ oxides for use in pseudocapacitors.

To gain additional insight into the storage mechanism of the $2 \mathrm{D} h-\mathrm{MoO}_{3}$ electrode, a peak shift of around $0.1 \mathrm{~V}$ was studied at sweep rates ranging from 2 to $100 \mathrm{mV} \mathrm{s}^{-1}$ (Fig. 5a). This peak shift indicated that the $2 \mathrm{D} h-\mathrm{MoO}_{3}$ was involved in the surfacecontrolled capacitive behaviour ${ }^{42}$. In addition, the relationship between the sweep rate and peak current was also analysed based on the following equation:

$$
i=a v^{b},
$$

where $i$ is the peak current (A), $v$ is the sweep rate $\left(\mathrm{mV} \mathrm{s}^{-1}\right)$ and $a$ and $b$ are coefficients ${ }^{39}$. The process is diffusion controlled (battery behaviour) when $b=0.5$ and is capacitive when $b=1$. The $b$ values for both the cathodic and anodic peaks were calculated to be 0.9 , further supporting the pseudocapacitive storage mechanism (Fig. 5d) that results from the fast and reversible redox reactions of $2 \mathrm{D} h-\mathrm{MoO}_{3}$ with minimal restriction of the ion diffusion. Therefore, a high capacity of $996 \mathrm{Cg}^{-1}$ was obtained at $2 \mathrm{mV} \mathrm{s}^{-1}$. Electrochemical impedance spectroscopy was also performed, and the results are shown in Fig. 5e. No apparent charge transfer resistance was observed, which indicates a combination of excellent ionic and electronic conductivities in the restacked $2 \mathrm{D} h-\mathrm{MoO}_{3}$ electrodes. The cycling stability of the pseudocapacitor was satisfactory. The initial capacitance increased because of the infiltration of the electrolyte, and $92 \%$ of the volumetric capacitance at a sweep rate of $10 \mathrm{mV} \mathrm{s}^{-1}$ was maintained after 6,000 cycles (Fig. 5f), which can be further improved by optimizing the electrode architecture. On the basis of the pseudocapacitive mechanism (no phase transformation), these materials are expected to exhibit good cycling stability that exceeds that of Li-ion batteries and simultaneously achieve high power-handling properties and fast charging time.

\section{Discussion}

The most significant result of this work is the demonstrated ability to generate high-quality $2 \mathrm{D}$ oxides with various compositions. The successful synthesis of various $2 \mathrm{D}$ binary transition metal oxides, combined with their outstanding performance in pseudocapacitor applications, will promote the exploration of $2 \mathrm{D}$ oxides for energy storage applications and beyond. In addition, achieving a combination of high gravimetric and volumetric capacitances is important for many applications, such as personal electronics, where the volume is limited. In this study, the volumetric capacity of $2 \mathrm{D} h-\mathrm{MoO}_{3}$ was as high as $1,100 \mathrm{Ccm}^{-3}$ at $2 \mathrm{mV} \mathrm{s}^{-1}$, which further indicates the advantage of the $2 \mathrm{D}$ morphology. Moreover, in order to compare our results with published data for other materials, the volumetric capacitance and energy/power density of the 2D $h-\mathrm{MoO}_{3}$ in a $\mathrm{Li}$-ion electrolyte are plotted in Supplementary Fig. 23b,c. The highest capacitance value was $550 \mathrm{~F} \mathrm{~cm}^{-3}$, exceeding that for $\mathrm{MoS}_{2}$ in $0.5 \mathrm{M}$ 1-ethyl-3-methylimidazolium tetrafluoroborate (EMIM $\left.\mathrm{BF}_{4}\right) / \mathrm{MeCN}\left(\sim 250 \mathrm{~F} \mathrm{~cm}^{-3}\right)^{11}$. In addition, the highest volumetric energy density was $270 \mathrm{mWh} \mathrm{cm}^{-3}$ with a power density of $1 \mathrm{~W} \mathrm{~cm}^{-3}$.

In conclusion, four transition metal oxides with non-layered structures were successfully synthesized with $2 \mathrm{D}$ morphologies using salt-assisted templating. On the basis of our experimental 
observations, we propose that the lateral growth of the $2 \mathrm{D}$ oxides was guided by the salt crystal geometry and promoted by lattice matching, and the thickness was restrained by the raw material supply (controlled amount of the dilute solution). The outstanding pseudocapacitive performance of $2 \mathrm{D} h-\mathrm{MoO}_{3}$ indicates the importance of the $2 \mathrm{D}$ morphology in energy storage. This material achieved $300 \mathrm{~F} \mathrm{~cm}^{-3}$ at $5 \mathrm{mV} \mathrm{s}^{-1}$ in the $\mathrm{Al}_{2}\left(\mathrm{SO}_{4}\right)_{3}$ electrolyte, which is favourable for the potential use of 2D oxides in Al-ion batteries. For organic electrolytes, capacities as high as $996 \mathrm{Cg}^{-1}(1.49 \mathrm{Li} / \mathrm{Mo})$ and $1,100 \mathrm{C} \mathrm{cm}^{-3}$ were obtained at a sweep rate of $2 \mathrm{mV} \mathrm{s}^{-1}$ owing to fast and reversible redox storage, resulting from fast ion diffusion and electron transport in the restacked $2 \mathrm{D} h-\mathrm{MoO}_{3}$ electrodes. Furthermore, the as-synthesized 2D oxides may find applications beyond energy storage, and this synthetic method could potentially be extended to materials other than oxides.

\section{Methods}

Synthesis of 2D $\mathbf{h}-\mathbf{M o O}_{\mathbf{3}}$ and $\mathbf{M o O}_{\mathbf{2}}$. In total, $0.1 \mathrm{~g}$ of Mo powder was dispersed in $20 \mathrm{ml}$ of ethanol with vigorous stirring for $30 \mathrm{~min}$. Then, $0.35 \mathrm{ml}$ of a $\mathrm{H}_{2} \mathrm{O}_{2}$ (30\%) solution was slowly added to the Mo powder suspension, which resulted in a blue solution after stirring for $18 \mathrm{~h}$. Then, the precursor solution was mixed with $320 \mathrm{~g}$ of $\mathrm{NaCl}$ powder followed by drying at $65^{\circ} \mathrm{C}$ with stirring. For $h-\mathrm{MoO}_{3}$, the as-obtained mixture was further annealed at $280^{\circ} \mathrm{C}$ for $1 \mathrm{~h}$ at a ramp rate of $2^{\circ} \mathrm{C} \mathrm{min}-1$ under an $\mathrm{Ar}$ atmosphere. After being cooled, the product was washed with deionized water to remove the $\mathrm{NaCl}$ templates and dispersed in ethanol for further characterization. For $\mathrm{MoO}_{2}$, the same procedure was applied, but the annealing temperature was $550^{\circ} \mathrm{C}$

Synthesis of 2D MnO. In total, $0.5 \mathrm{~g} \mathrm{Mn}\left(\mathrm{CH}_{3} \mathrm{COO}\right)_{2} \cdot 4 \mathrm{H}_{2} \mathrm{O}$ was dispersed in $25 \mathrm{ml}$ of ethanol with stirring for $5 \mathrm{~min}$ to obtain the $\mathrm{MnO}$ precursor solution. Then, the precursor solution was mixed with $400 \mathrm{~g}$ of potassium chloride power and dried at $70^{\circ} \mathrm{C}$ with stirring. The as-obtained mixture was further annealed at $400^{\circ} \mathrm{C}$ for $1 \mathrm{~h}$ at a ramp rate of $2^{\circ} \mathrm{C} \mathrm{min}^{-1}$ under an $\mathrm{Ar}$ atmosphere. After being cooled, the product was washed with deionized water to remove the $\mathrm{KCl}$ templates and dispersed in ethanol for further characterization.

Synthesis of 2D $\boldsymbol{h}$-WO $\mathbf{W}_{3}$. In total, $0.1 \mathrm{~g}$ of tungsten powder was dispersed in $20 \mathrm{ml}$ of ethanol with vigorous stirring for $30 \mathrm{~min}$. Then, $1 \mathrm{ml}$ of a $\mathrm{H}_{2} \mathrm{O}_{2}(30 \%)$ solution was slowly added to the $\mathrm{W}$ powder suspension, which produced a white solution after stirring for $36 \mathrm{~h}$. Then, the precursor solution was mixed with $400 \mathrm{~g}$ of $\mathrm{KCl}$ powder followed by drying at $70^{\circ} \mathrm{C}$ with stirring. The as-obtained mixture was further annealed at $500^{\circ} \mathrm{C}$ for $1 \mathrm{~h}$ using a ramp rate of $2^{\circ} \mathrm{C} \mathrm{min}-1$ under an $\mathrm{Ar}$ atmosphere. After being cooled, the product was washed with deionized water to remove $\mathrm{KCl}$ and dispersed in ethanol for further characterization.

Preparation of electrodes. The vacuum-assisted filtration method was used to fabricate the multilayer electrodes. The as-produced 2D oxide/ethanol dispersion was filtered through a membrane (Celgard, USA). The obtained film was dried at room temperature and then punched into circular electrodes. The thickness of the electrodes was $1 \mu \mathrm{m}$. The density of each electrode was $\sim 1.1 \mathrm{~g} \mathrm{~cm}^{-3}$.

Characterization. The morphology and structure of the samples were studied using field-emission SEM (FE-SEM, FEI Nova 450 Nano), optical microscopy (Nikon), high-resolution transmission electron microscopy (HRTEM, TECNAI), AFM (Shimadzu), X-ray diffraction (X’Pert Pro, PANanalytical), XPS (ESCALab250), Raman spectroscopy and photoluminescence (LabRAM HR800). For the optical microscopy and AFM measurements, the samples were dropped on $\mathrm{SiO}_{2} / \mathrm{Si}$ and dried at room temperature. The electron conductivity was measured using a Keithley 4,200. All of the electrochemical tests were carried out using ECLab and CHI660E. For the typical three-electrode set-up, $\mathrm{Ag} / \mathrm{AgCl}$ (CHI, USA) was used as the reference electrode, YP-50 (Kuraray, Japan) was used as the counter electrode and a Celgard film served as the separator (Celgard, USA). All of the tests were conducted in Swagelok cells (Swagelok, USA). The electrochemical impedance was measured from 1 to $1 \mathrm{MHz}$ with a potential amplitude of $10 \mathrm{mV}$. The organic electrolyte was fabricated by dissolving $\mathrm{LiClO}_{4}$ in ethylene carbonate/dimethyl carbonate at a concentration of $1 \mathrm{M}$.

\section{References}

1. Simon, P., Gogotsi, Y. \& Dunn, B. Where do batteries end and supercapacitors begin? Science 343, 1210-1211 (2014).

2. Augustyn, V., Simon, P. \& Dunn, B. Pseudocapacitive oxide materials for highrate electrochemical energy storage. Energy Environ. Sci. 7, 1597-1614 (2014).
3. Sun, W. M. et al. High surface area tunnels in hexagonal $\mathrm{WO}_{3}$. Nano Lett. 15, 4834-4838 (2015).

4. Mai, L. Q. et al. Synergistic interaction between redox-active electrolyte and binder-free functionalized carbon for ultrahigh supercapacitor performance. Nat. Commun. 4, 2923 (2013).

5. Lu, X. et al. Improving the cycling stability of metal-nitride supercapacitor electrodes with a thin carbon shell. Adv. Energy Mater. 4, 1300994-1300999 (2013).

6. Béguin, F., Presser, V., Balducci, A. \& Frackowiak, E. Carbons and electrolytes for advanced supercapacitors. Adv. Mater. 26, 2219-2251 (2014).

7. Sun, G. et al. Hybrid fibers made of molybdenum disulfide, reduced graphene oxide, and multi-walled carbon nanotubes for solid-state, flexible, asymmetric supercapacitors. Angew. Chem. Int. Ed. 54, 4651-4656 (2015).

8. Yang, X., Cheng, C., Wang, Y., Qiu, L. \& Li, D. Liquid-mediated dense integration of graphene materials for compact capacitive energy storage. Science 341, 534-537 (2013).

9. Ghidiu, M., Lukatskaya, M. R., Zhao, M. Q., Gogotsi, Y. \& Barsoum, M. W. Conductive two-dimensional titanium carbide 'clay' with high volumetric capacitance. Nature 516, 78-81 (2014).

10. Lukatskaya, M. R. et al. Cation intercalation and high volumetric capacitance of two-dimensional titanium carbide. Science 341, 1502-1505 (2013).

11. Acerce, M., Voiry, D. \& Chhowalla, M. Metallic $1 \mathrm{~T}$ phase $\mathrm{MoS}_{2}$ nanosheets as supercapacitor electrode materials. Nat. Nanotechnol. 10, 313-318 (2015).

12. Nicolosi, V., Chhowalla, M., Kanatzidis, M. G., Strano, M. S. \& Coleman, J. N. Liquid exfoliation of layered materials. Science 340, 1420-1438 (2013).

13. Conway, B. E., Birss, V. \& Wojtowicz, J. The role and utilization of pseudocapacitance for energy storage by supercapacitors. J. Power Sources $\mathbf{6 6}$, 1-14 (1997).

14. Liu, Z., Xu, K., Sun, H. \& Yin, S. One-step synthesis of single-layer $\mathrm{MnO}_{2}$ nanosheets with multi-role sodium dodecyl sulfate for high-performance pseudocapacitors. Small 11, 2182-2191 (2015).

15. Hanlon, D. et al. Production of molybdenum trioxide nanosheets by liquid exfoliation and their application in high-performance supercapacitors. Chem. Mater. 26, 1751-1763 (2014)

16. Lee, Y. H. et al. Synthesis of large-area $\mathrm{MoS}_{2}$ atomic layers with chemical vapor deposition. Adv. Mater. 24, 2320-2325 (2012).

17. Li, X. et al. Large-area graphene single crystals grown by low-pressure chemical vapor deposition of methane on copper. J. Am. Chem. Soc. 133, 2816-2819 (2011).

18. van der Zande, A. M. et al. Grains and grain boundaries in highly crystalline monolayer molybdenum disulphide. Nat. Mater. 12, 554-561 (2013).

19. Coleman, J. N. et al. Two-dimensional nanosheets produced by liquid exfoliation of layered materials. Science 331, 568-571 (2011).

20. Paton, K. R. et al. Scalable production of large quantities of defect-free few-layer graphene by shear exfoliation in liquids. Nat. Mater. 13, 624-630 (2014).

21. Zheng, J. et al. High yield exfoliation of two-dimensional chalcogenides using sodium naphthalenide. Nat. Commun. 5, 2995 (2014).

22. Huang, X. et al. Freestanding palladium nanosheets with plasmonic and catalytic properties. Nat. Nanotechnol. 6, 28-32 (2011).

23. Du, Y. et al. A general method for the large-scale synthesis of uniform ultrathin metal sulphide nanocrystals. Nat. Commun. 3, 1177 (2012).

24. Son, J. S. et al. Large-scale soft colloidal template synthesis of $1.4 \mathrm{~nm}$ thick CdSe nanosheets. Angew. Chem. Inter. Ed. 121, 6993-6996 (2009).

25. Sun, Z. et al. Generalized self-assembly of scalable two-dimensional transition metal oxide nanosheets. Nat. Commun. 5, 3813 (2014).

26. Tan, C. \& Zhang, H. Wet-chemical synthesis and applications of non-layer structured two-dimensional nanomaterials. Nat. Commun. 6, 7873 (2015)

27. Xu, M., Liang, T., Shi, M. \& Chen, H. Graphene-like two-dimensional materials. Chem. Rev. 113, 3766-3798 (2013).

28. Liu, R. et al. Sodium chloride template synthesis of cubic tin dioxide hollow particles for lithium ion battery applications. ACS Appl. Mater. Interfaces 4, 1537-1542 (2012).

29. Wang, B. et al. Synthesis of $\mathrm{NaCl}$ single crystals with defined morphologies as templates for fabricating hollow nano/micro-structures. RSC Adv. 5, 5072-5076 (2015).

30. Li, N. et al. Free-standing and transparent graphene membrane of polyhedron box-shaped basic building units directly grown using a nacl template for flexible transparent and stretchable solid-state supercapacitors. Nano Lett. 15, 3195-3203 (2015).

31. Tang, Y. et al. Efficient Ag@AgCl cubic cage photocatalysts profit from ultrafast plasmon-induced electron transfer processes. Adv. Func. Mater. 23, 2932-2940 (2013).

32. Takenaka, S., Miyake, S., Uwai, S., Matsune, H. \& Kishida, M. Preparation of metal oxide nanofilms using graphene oxide as a template. J. Phys. Chem. C 119, 12445-12454 (2015)

33. Lee, J. M., Kim, I. Y., Han, S. Y., Kim, T. W. \& Hwang, S. J. Graphene nanosheets as a platform for the $2 \mathrm{D}$ ordering of metal oxide nanoparticles: 
mesoporous 2D aggregate of anatase $\mathrm{TiO}_{2}$ nanoparticles with improved electrode performance. Chemistry 18, 13800-13809 (2012).

34. Cao, H., Zhou, X., Zheng, C. \& Liu, Z. Two-dimensional porous micro/nano metal oxides templated by graphene oxide. ACS Appl. Mater. Interfaces 7, 11984-11990 (2015).

35. Kuykendall, T. et al. Crystallographic alignment of high-density gallium nitride nanowire arrays. Nat. Mater. 3, 524-528 (2004).

36. Lee, W. C. et al. Graphene-templated directional growth of an inorganic nanowire. Nat. Nanotechnol. 10, 423-428 (2015).

37. Zheng, L., Xu, Y., Jin, D. \& Xie, Y. Novel metastable hexagonal $\mathrm{MoO}_{3}$ nanobelts: synthesis, photochromic, and electrochromic properties. Chem. Mater. 21, 5681-5690 (2009).

38. Tsumura, T. \& Inagaki, M. Lithium insertion/extraction reaction on crystalline $\mathrm{MoO}_{3}$. Solid State Ionics 104, 183-189 (1997).

39. Augustyn, V. et al. High-rate electrochemical energy storage through $\mathrm{Li}^{+}$ intercalation pseudocapacitance. Nat. Mater. 12, 518-522 (2013).

40. Lin, M. C. et al. An ultrafast rechargeable aluminium-ion battery. Nature $\mathbf{5 2 0}$, 325-328 (2015)

41. Brezesinski, T., Wang, J., Tolbert, S. H. \& Dunn, B. Ordered mesoporous $\alpha$ $\mathrm{MoO}_{3}$ with iso-oriented nanocrystalline walls for thin-film pseudocapacitors. Nat. Mater. 9, 146-151 (2010).

42. Conway, B. E. Transition from 'supercapacitor' to 'battery' behavior in electrochemical energy storage. J. Electrochem. Soc. 138, 1539-1548 (1991).

\section{Acknowledgements}

This work was financially supported by the National Natural Science Foundation of China (grants 51322210, 61434001, 61274055 and 61322401) and Director Fund of WNLO. J.Z. wishes to thank the National Program for Support of Top-notch Young Professionals for financial support. J.T. wishes to thank the National 1,000 Young Talents project for financial support. Y.G. was supported by the US Department of Energy, Office of Electricity, Energy Storage Systems Program through Sandia National Laboratory. We wish to thank Kathleen Maleski at Drexel University for helpful comments on the manuscript. We also wish to thank Dr D. Yong and Professor Z.L. Wang from Georgia
Institute of Technology for helpful discussions on the growth mechanism. In addition, we wish to thank facility support from the Center for Nanoscale Characterization \& Devices, WNLO of Huazhong University of Science and Technology (HUST) and the Analytical and Testing Center of HUST.

\section{Author contributions}

J.Z. and J.T. conceived the idea. X.X., H.S., J.Z., J.T. and Y.G. designed the experiments. X.X., H.S., S.L., X.Z. and J.S. carried out the experiments. X.X., H.S., J.Z., J.T., and Y.G. analysed the data. Y.Z. and B.Y. assisted in analysis. Z.H. assisted with the X-ray diffraction measurement. T.L. assisted in the analysis of the electrochemical performance. Q.Z. assisted with the AFM measurements. L.J. provided helpful suggestions for analysis and manuscript revision. X.X., H.S., J.Z., J.T. and Y.G. wrote the manuscript. All of the authors commented on the manuscript.

\section{Additional information}

Supplementary Information accompanies this paper at http://www.nature.com/ naturecommunications

Competing financial interests: The authors declare no competing financial interests.

Reprints and permission information is available online at http://npg.nature.com/ reprintsandpermissions/

How to cite this article: Xiao, X. et al. Scalable salt-templated synthesis of twodimensional transition metal oxides. Nat. Commun. 7:11296 doi: 10.1038/ncomms11296 (2016).

(c) (i) This work is licensed under a Creative Commons Attribution 4.0 International License. The images or other third party material in this article are included in the article's Creative Commons license, unless indicated otherwise in the credit line; if the material is not included under the Creative Commons license, users will need to obtain permission from the license holder to reproduce the material. To view a copy of this license, visit http://creativecommons.org/licenses/by/4.0/ 